\title{
IMPACTO DE MÉTODOS NATURAIS PARA CONSERVAÇÃO DE ÁGUA E SOLO NO SEMIÁRIDO BRASILEIRO
}

\author{
Montenegro, A.A.A. ${ }^{1}$; Lopes, I. ${ }^{2}$; AlmeidA, T.A.B. ${ }^{1 ;}$ \\ Lima, J.L.M.P. DE ${ }^{3}$; Montenegro, H.G.L.A. ${ }^{1} \&$ ARAúJo, B.G. ${ }^{1}$
}

\begin{abstract}
RESUMO
As técnicas conservacionistas são altamente relevantes para o uso sustentável do solo, especialmente em regiões do semiárido. O objetivo deste estudo foi investigar o impacto de técnicas conservacionistas na redução das perdas de sedimentos e no incremento da umidade do solo, utilizando parcelas sob chuva natural. O estudo foi realizado na Bacia do Rio Alto Ipanema, Brasil. O monitoramento experimental foi realizado em nove parcelas com diferentes condições de cobertura (solo descoberto; cobertura natural; cobertura morta; Palma Forrageira). A cobertura morta apresentou desempenho semelhante à cobertura vegetal arbórea natural, para controle de escoamento e perdas de sedimentos. A umidade do solo foi significativamente maior nas parcelas com cobertura morta do que nas parcelas de solo descoberto. Adicionalmente, as linhas de Palma produziram resultados hidrológicos promissores. Experimentos complementares em laboratório, com chuvas simuladas, apresentaram resultados consistentes com as observações em campo, sob chuva natural.
\end{abstract}

Palavras-chave: cobertura morta, cordões de Palma Forrageira, práticas naturais, técnicas conservacionistas.

\begin{abstract}
Impact of nature-based solutions for soil and water conservation in semiarid Brazil.

Conservation techniques are highly relevant, for sustainable land use, especially in semiarid regions. The aim of this study was to investigate the impact of conservation techniques in reducing sediment losses and increasing soil moisture, under natural rainfall in soil erosion plots. The

1.- Departamento de Engenharia Agrícola, Universidade Federal Rural de Pernambuco, Rua Dom Manuel de Medeiros, s/n, Dois Irmãos, CEP 52171-900, Recife- Pernambuco, Brasil. abelardo.montenegro@ufrpe.br 2.- Departamento de Engenharia Agronômica, Instituto Federal Baiano, Campus Bom Jesus da Lapa, BR-349, s/n, Zona Rural, CEP 47600-000, Bom Jesus da Lapa - Bahia, Brasil. iug.lopes@ifbaiano.edu.br

3.- Departamento de Engenharia Civil, Centro de Ciências do Mar e do Ambiente, Faculdade de Ciências e Tecnologias da Universidade de Coimbra, Rua Luís Reis Santos - Pólo II da Universidade de Coimbra, 3030788 Coimbra, Portugal. plima@dec.uc.pt

Manuscrito recibido el 15 de mayo de 2020 y aceptado para su publicación el 28 de septiembre de 2020 .
\end{abstract}

Montenegro, A.A.A.; Lopes, I.; Almeida, T.A.B.; Lima, J.L.M.P. de; Montenegro, H.G.L.A. \& Araújo, B.G. Impacto de métodos naturais para conservação de água e solo no semiárido brasileiro. FAVE - Ciencias Agrarias 19 (2): 45-59. CC BY-NC-SA 4.0 


\section{A.A.A. Montenegro et al.}

study was carried out in the Alto Ipanema River Basin, Brazil. The experimental monitoring was carried out in nine plots with different cover conditions (bare soil; natural cover; mulch; Forrage Palm Cactus). Mulch presented similar performance than natural vegetation cover, to control runoff and sediment losses. Soil moisture was significantly higher in plots with mulch when compared with in those with bare soil. Additionally, Palma lines produced promising hydrological results. Complementary experiments in the laboratory, with simulated rainfall, were consistent with field observations under natural events.

Key words: mulch, Forage Palm lines, nature-based solutions, conservation practices.

\section{INTRODUÇÃO}

O solo é um componente essencial e multifuncional do ecossistema terrestre, fornecendo bases para produção de alimentos, controlando processos hidrológicos, e oferecendo serviços ecossistêmicos, cruciais para se alcançar os Objetivos de Desenvolvimento Sustentável (ODS) estabelecidos pelas Nações Unidas no tocante à produção de alimentos e qualidade de vida (Darkwah et al., 2019; Hou et al., 2020). A redução da qualidade do solo, resultante de sua degradação, diminui a sua produtividade, comprometendo a sustentabilidade da agricultura, a qualidade e a estabilidade do meio ambiente, e a disponibilidade de recursos hídricos de qualidade nas bacias hidrográficas, causando consequentemente um efeito adverso no desenvolvimento socioeconômico e sustentável. A região semiárida Brasileira apresenta limitada disponibilidade de recursos hídricos e elevada susceptibilidade à ocorrência de erosão, devido a quatro fatores preponderantes: solos rasos com baixa capacidade de retenção de água; regime pluviométrico com distribuição espacial e temporal irregular, ocorrendo com frequência chuvas de alta intensidade; elevadas temperaturas, que estão associadas a altas taxas de evapotrans- piração; e limitada cobertura vegetal, com predomínio de espécies caducifólias.

Importantes discussões referentes à conservação do solo estão presentes no relatório do IPCC ("Intergovernmental Panel on Climate Change"), sobre mudanças climáticas, desertificação, degradação e manejo sustentável do solo, segurança alimentar, e fluxos de gases de efeito estufa em ecossistemas terrestres (Shukla et al., 2019). O relatório destaca que a intensificação em mudanças de uso e ocupação de terra associada às mudanças climáticas contribuem para processos de degradação e desertificação do solo. Além disso, é relatado que manejos inadequados de solos podem intensificar períodos de seca. Nesse sentido, o relatório recomenda diversas técnicas conservacionistas que podem ser adotadas potencial de evitar, reduzir e até reverter diversos processos de degradação causados pela interação dos fatores naturais (solo, clima, relevo) e antrópicos. Além disso, as técnicas conservacionistas proporcionam condições favoráveis para produção agrícola, no tocante ao suprimento de água e nutriente, em especial nos períodos secos que tornam-se maiores e mais intensos devido às mudanças climáticas. Dentre as alternativas naturais de conservação de água e solo adaptáveis às bacias hidrográficas do 
semiárido, e com baixo custo, destacam-se a cobertura morta com restos vegetais (Santos et al., 2008; Lopes et al., 2019), e os cordões vegetativos como barramentos vegetais, utilizando por exemplo a Palma Forrageira (Opuntia sp.) (Borges et al., 2014; Montenegro et al., 2019). Esses estudos vêm sendo sistematicamente realizados no Estado de Pernambuco, na Bacia Representativa do Alto Ipanema, como parte das ações experimentais da Rede de Hidrologia do Semiárido (REHISA), e mais recentemente do Consórcio Universitas, este último reunindo universidades públicas de Pernambuco em prol do incremento da segurança hídrica no semiárido de Pernambuco.

A cobertura morta com restos vegetais é largamente difundida, sendo capaz de amortecer os impactos das gotas de chuva, incrementar a infiltração, e reduzir a erosão hídrica (Sarkar \& Singh, 2007; Montenegro et al., 2013; Cerdà et al., 2017; Prats et al, 2017; Flores et al., 2019). Jordán et al. (2010) verificaram que aplicações de cobertura morta por longos períodos melhoraram as propriedades físicas e químicas de solos do semiárido da Espanha. Aumentos na interceptação da chuva, no atraso na geração de escoamento, e controles de erosão ocorreram com coberturas mortas de palha de trigo seca com densidade de cobertura de 5 $\mathrm{Mg} \mathrm{ha}^{-1}$ por ano. Já quanto à adoção de cordões vegetativos, vários estudos foram realizados para avaliar o potencial e os efeitos da adoção desta técnica, dentre eles Pan et al. (2018) e Haddaway et al. (2018), este último realizando vasta revisão das aplicações dos cordões vegetativos para a proteção ambiental. A adoção de cordões vegetativos requer a utilização de plantas adaptadas às condições climáticas locais. No caso de regiões semiáridas, a babosa (Aloe Vera (L).)
(Varela et al., 2014) e a Palma Forrageira (Opuntia sp.) (Montenegro et al., 2019) são alternativas de plantas que podem ser utilizadas nos cordões vegetativos.

Desse modo, o objetivo deste artigo é avaliar o efeito de diferentes técnicas naturais de conservação sobre a erosão hídrica e o teor de água no solo, na Bacia do Alto Ipanema, semiárido de Pernambuco, complementados com avaliações em laboratório.

\section{MATERIAIS E MÉTODOS}

As investigações hidrológicas vêm sendo desenvolvidas na Bacia Representativa do Alto Ipanema, desde 2009. Esta bacia está localizada no município de Pesqueira, no semiárido do Estado de Pernambuco, Brasil (Figura 1), situada entre as coordenadas $8^{\circ} 34^{\prime} 17^{\prime \prime}$ e $8^{\circ} 18^{\prime} 11^{\prime \prime}$ de Latitude Sul, e $37^{\circ} 1^{\prime} 35^{\prime \prime}$ e $36^{\circ} 47^{\prime} 20^{\prime}$ " de Longitude Oeste. O clima da região segundo a classificação de Köppen, é do tipo BSsh (extremamente quente, semiárido). A precipitação média anual, conforme dados históricos locais (1910 a 2012) é de 671,9 mm. De acordo com os dados normais climatológicos, a evaporação total anual é de $1.589,80 \mathrm{~mm}$, a temperatura média de $24,7{ }^{\circ} \mathrm{C}$, com máxima de $29,0{ }^{\circ} \mathrm{C}$ e mínima de $18,5{ }^{\circ} \mathrm{C}$, respectivamente. A vegetação predominante é a Caatinga hipoxerófila, cactáceas e bromeliáceas (Montenegro e Montenegro, 2004), com comportamento caducifólio.

Três diferentes sítios experimentais foram implantados, cada um constando de cinco parcelas com 4,5 m de largura por 11 $\mathrm{m}$ de comprimento, delimitadas por alvenaria (Figura 2). As parcelas experimentais 


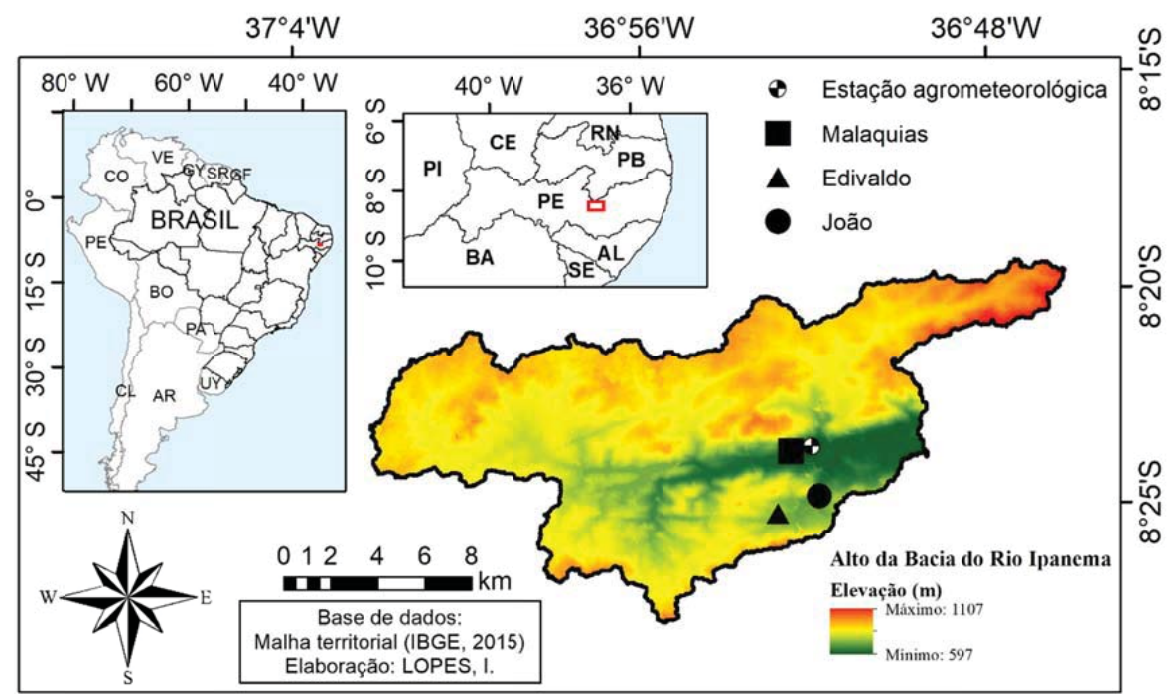

Figura 1- Localização da bacia hidrográfica de estudo, no semiárido de Pernambuco, Brasil. Fonte: Lopes et al. (2019).

Figure 1-Location of the study basin in the semiarid region of Pernambuco, Brazil. Source: Lopes et al. (2019).

encontram-se equipadas com sondas TDR (Reflectometria no Domínio do Tempo) para medição da umidade do solo, além de tubos de acesso em PVC para medições com Sondas de Nêutrons. As equações de calibração para Sonda de Nêutrons, na camada de $0-0,2 \mathrm{~m}$ e $0,2-0,4 \mathrm{~m}$, e para TDR na camada de 0-0,20 m apresentam comportamento linear, com coeficientes angulares de $0,345,0,4106,0,002$, coeficientes lineares de $0,002,0,0227$ e $-0,0431$, e coeficientes de determinação de $0,98,0,99$ e 0,90 , respectivamente. Essas equações permitem a obtenção da umidade gravimétrica a partir das leituras dos equipamentos. Cada sítio possui um pluviógrafo automático de báscula, modelo TB4L da Campbell Scientific. Em dois sítios, o solo foi classificado como Argissolo Amarelo Eutrófico típico, e em um sítio, o solo foi classificado como Neossolo Regolítico. As características físicas dos solos foram descritas por Santos (2010). Em cada experimento foram utilizados os seguintes tratamentos: cobertura natural $(\mathrm{PCN})$ composta por caatinga rasa de pequenos e médio porte, solo descoberto (PSD), cordão vegetativo de Palma Forrageira $(\mathrm{PF})$ planta em espaçamento regular de $0,5 \times 1,5 \mathrm{~m}$, cobertura morta de pó de coco com densidade de $8 \mathrm{Mg} \mathrm{ha}^{-1}$ (PCM).

Experimentos adicionais foram realizados em laboratório com simulador de chuva, comparando-se o desempenho da cobertura morta de pó de coco a $8 \mathrm{Mg} \mathrm{ha}^{-1}$ (PCM) e barramento com Palma Forrageira (PF), em relação ao solo descoberto (PSD). Uti- 

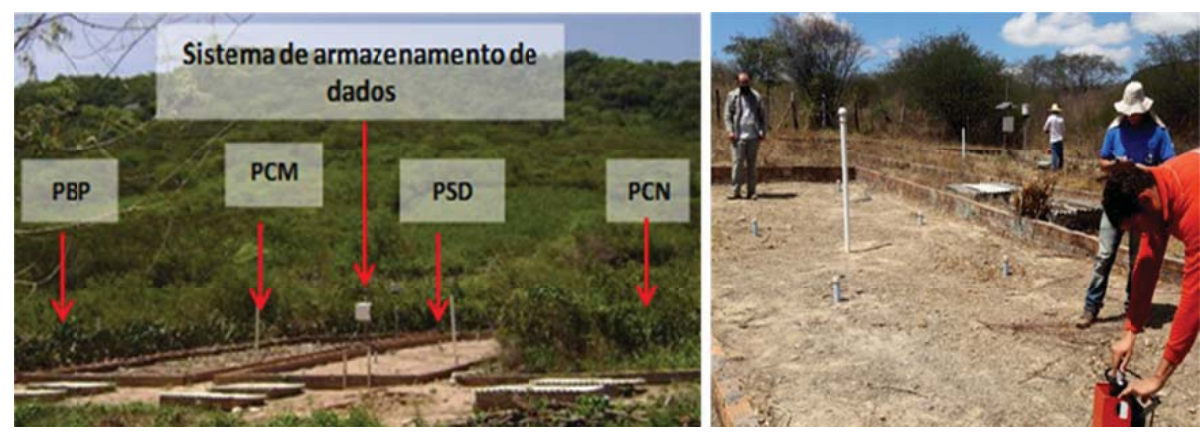

Figura 2- Vista das parcelas experimentais com diferentes condições de tratamentos, na estação chuvosa, e monitoramento com Sonda de Nêutrons na parcela descoberta, na estação seca.

Figure 2- View of experimental plots with different treatment conditions, in the rainy season, and monitoring with Neutron Probe at the bare soil plot, in the dry season.

lizou-se uma parcela experimental de 1,51 $\times 0,51 \mathrm{~m}$, com uma declividade de $10 \%$, preenchida com o solo correspondente da bacia do Alto Ipanema-PE (Argissolo Amarelo Eutrófico típico), Brasil. A intensidade de chuva e sua duração foi adotada a partir da equação da intensidade-duração-frequência (IDF) da precipitação para tempo de retorno de 7 anos, para o município de Pesqueira-PE, segundo Coutinho et al. (2010).

Para os tratamentos foi aplicada uma chuva simulada de intensidade uniforme de $90 \mathrm{~mm} \mathrm{~h}^{-1}$ durante 15 minutos, utilizando-se um simulador de chuva de intensidade variável, onde para cada tratamento foram realizadas três repetições de simulação de chuva. A partir do início do escoamento superficial foram efetivadas as coletas dos volumes escoados a com intervalos regulares, seguindo o procedimento descrito por Cogo (1978). A lâmina escoada foi calculada com base no volume coletado da enxurrada por unidade de área na parcela de erosão. Para medição da umidade do solo em laboratório foram utilizados quatro sensores de umidade FALKER HidroFarm HFM 1010, distribuídos no sentido longitudinal do declive a partir da seção inicial da parcela, considerando o percentual em relação à saturação. A umidade foi verificada antes e após cada simulação, verificando a umidade antecedente à cada ensaio.

$\mathrm{O}$ delineamento experimental nas parcelas foi o inteiramente casualizado e os dados experimentais foram submetidos à análise estatística clássica. Para comparação das médias de umidade dos tratamentos teste de Tukey foi aplicado ao nível de significância de 5\%, com auxílio do software livre RStudio. 


\section{RESULTADOS E DISCUSSÃO}

A Figura 3 apresenta o valor médio das medições de umidade do solo na camada de 0 a $20 \mathrm{~cm}$, no sítio experimental com Neossolo Regolítico, de 1 de janeiro de 2018 a 15 de fevereiro de 2019, totalizando 335 medições por tratamento, descontando-se as falhas de 11 de abril a 9 de maio e 15 de junho a 31 de julho de 2018. Na Figura 3 também estão apresentados os registros diários dos eventos de chuva. Em período chuvoso em 2018, verifica-se a pronunciada resposta da parcela com cobertura natural, com elevados picos de umidade, apresentando também uma desfasagem positiva na recessão, ou seja, um padrão de secamento mais lento que os demais tratamentos, apesar da maior transpiração da vegetação natural neste período. Este comportamento também foi verificado por Montenegro et al. (2019), para registros em meados de 2017, no mesmo sítio, estando associado à capacidade de armazenamento de água da cobertura vegetal, e ao fluxo pelo caule. Para o período seco, constata-se o adequado desempenho da cobertura morta na manutenção da umidade do solo, seguida pela técnica de barramento em Palma Forrageira.

Em relação ao tratamento com Palma, a adoção do cordão vegetativo mostrou-se promissor na manutenção das umidades, sendo uma técnica eficiente quando comparado ao PSD, com destaque para o período de julho de 2018 a dezembro de 2019, onde se aproximaram de PCN. Autores como Borges et al. (2014) e Montenegro

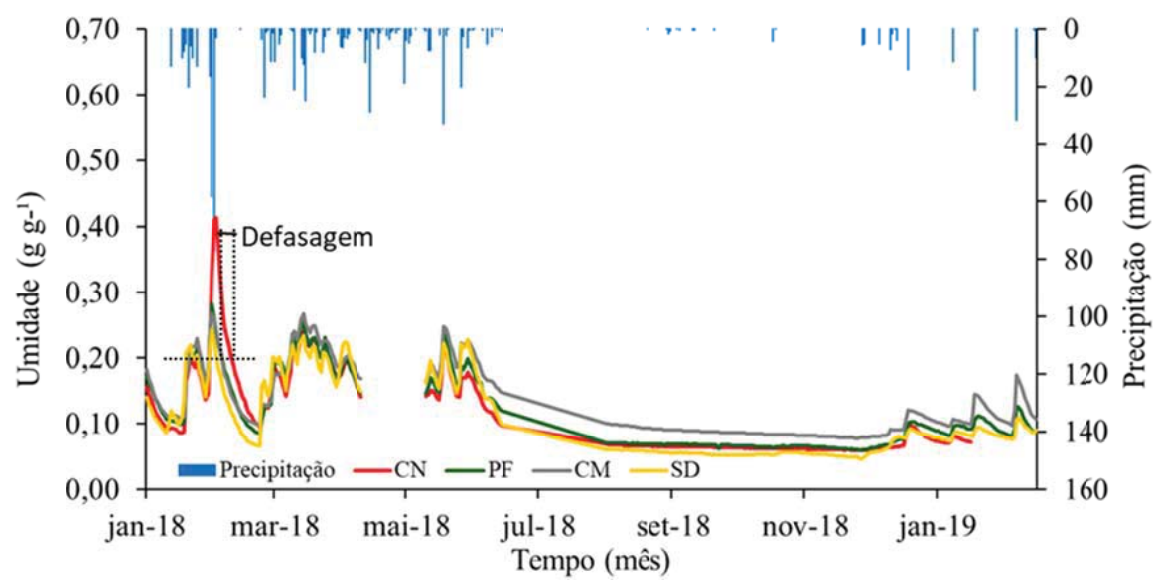

Figura 3- Distribuição temporal da precipitação e da umidade do solo nas parcelas com tratamento e cobertura natural (CN), Barramento com Palma Forrageira (PF), Cobertura morta de pó de coco (CM) e Solo descoberto (SD), de janeiro de 2018 a janeiro de 2019.

Figure 3- Temporal distribution of rainfall and soil moisture in plots with treatments of natural cover (CN), Forrage Palm (PF), Coconut powder mulch (CM) and bare soil (SD), from january 2018 to january 2019. 
et al. (2019) destacam o incremento no armazenamento de água no solo e a redução da taxa de erosão em solos cultivados com cordões vegetativos.

A Figura 4 apresenta as distribuições de frequência experimentais para as leituras de umidade nos tratamentos. Pode-se verificar que a utilização das práticas conservacionistas reflete em maior probabilidade de incremento de umidade no solo em comparação com o solo descoberto, com destaque para o tratamento de cobertura morta, que apresentou maior acumulo de água no perfil. Borges et al. (2014) ao utilizarem a cobertura morta de Capim Elefante (densidade de $7 \mathrm{Mg} \mathrm{ha}^{-1}$ ) associada a barramentos de pedra no cultivo de milho de sequeiro, nas mesmas parcelas experimentais, também relataram maior potencial de retenção de água no solo, além de incrementos significativos na produção.
Menezes et al. (2013), ao compararem o comportamento temporal da água no solo, na Bacia Representativa do Alto Ipanema, destacaram que solos descobertos apresentam menor umidade em comparação com solo com cobertura natural de vegetação de Caatinga, após eventos de chuva, constatando que na condição de solo exposto há maior escoamento superficial, promovendo maior erosão e selamento da superfície do solo. Entretanto, em períodos de estiagem, em que a umidade do solo se encontra em valores reduzidos, há maior perda de água em solos de vegetação natural de Caatinga, devido à evapotranspiração, que é consistente com os resultados apresentados na Figura 4.

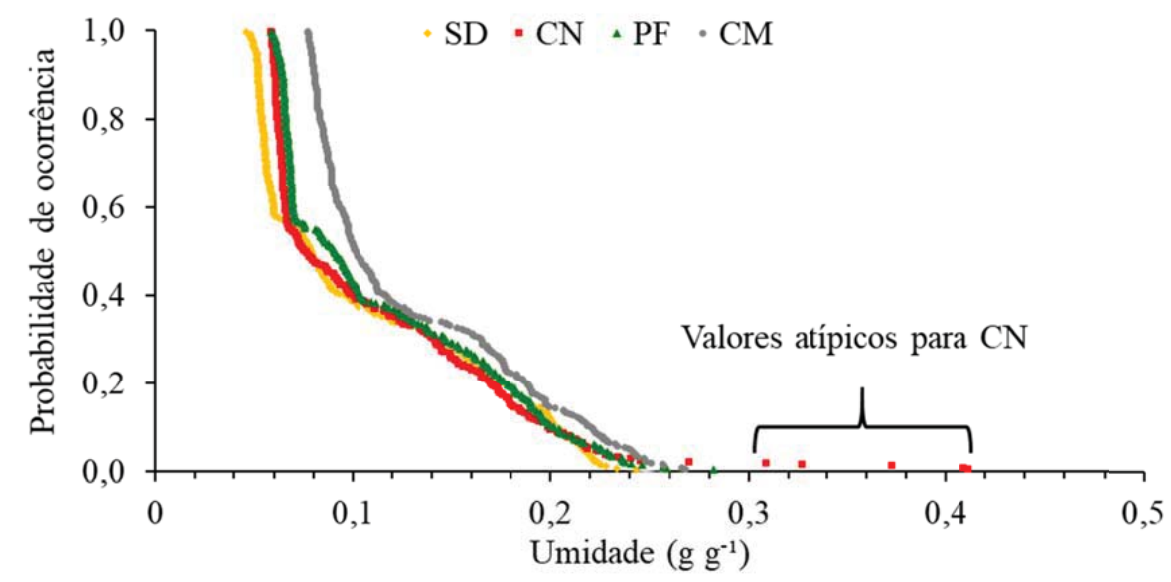

Figura 4- Distribuições de frequência experimentais das umidades do solo medidas com sondas TDR, de janeiro de 2018 a janeiro de 2019. Bacia do Alto Ipanema, Pernambuco, Brasil.

Figure 4- Experimental frequency distributions of soil moisture measured with TDR probes, from january 2018 to january 2019. Alto Ipanema Basin, Pernambuco, Brazil. 


\section{A.A.A. Montenegro et al.}

Na mesma área experimental, Montenegro et al. (2019) analisaram separadamente a distribuição de umidade do solo para os períodos úmidos e secos entre 2016 e 2018, e destacaram os benefícios de práticas vegetativas naturais tais como, (i) o aumento da infiltração e armazenamento de água em solos com cobertura natural (Caatinga) devido aos processos de interceptação nos períodos úmidos, ressaltando assim a importância das iniciativas de reflorestamento na área; e (ii) manutenção da umidade do solo nos períodos seco devido a redução das perdas de água do solo por evaporação utilizando cobertura morta de pó de coco.

Em relação ao uso da prática de cultivo Tukey) superiores aos das parcelas descode cordões de Palma, Lima et al. (2015a) bertas.

Tabela 1. Umidade volumétrica média do solo nos conjuntos experimentais para o período de janeiro de 2017 a janeiro de 2019, nas camadas de 0,0-0,2 m e 0,2-0,4 m.

Table 1. Mean soil volumetric moisture for the experimental plots at $0.0-0.2 \mathrm{~m}$ and $0.2-0.4 \mathrm{~m}$ layers.

\begin{tabular}{clcc}
\hline \multirow{2}{*}{ Local } & Tratamento* & \multicolumn{2}{c}{ Umidade volumétrica $\left(\mathrm{cm}^{-3} \mathrm{~cm}^{-3}\right)^{\star *}$} \\
& $0,0-0,2$ & $0,2-0,4$ \\
\hline \multirow{3}{*}{ Malaquias } & PCN & $0,101 \mathrm{~b}$ & $0,133 \mathrm{~b}$ \\
& PSD & $0,088 \mathrm{c}$ & $0,129 \mathrm{c}$ \\
& PCM & $0,104 \mathrm{a}$ & $0,144 \mathrm{a}$ \\
& PF & $0,112 \mathrm{a}$ & $0,145 \mathrm{a}$ \\
\hline \multirow{2}{*}{ Edivaldo } & PCN & $0,079 \mathrm{~b}$ & $0,141 \mathrm{~b}$ \\
& PSD & $0,077 \mathrm{c}$ & $0,137 \mathrm{c}$ \\
& PCM & $0,082 \mathrm{a}$ & $0,149 \mathrm{a}$ \\
& PF & $0,079 \mathrm{~b}$ & $0,142 \mathrm{ab}$ \\
\hline \multirow{3}{*}{ João } & PCN & $0,056 \mathrm{~b}$ & $0,104 \mathrm{~b}$ \\
& PSD & $0,049 \mathrm{c}$ & $0,097 \mathrm{c}$ \\
& PCM & $0,067 \mathrm{a}$ & $0,122 \mathrm{a}$ \\
& PF & $0,064 \mathrm{a}$ & $0,108 \mathrm{a}$ \\
\hline
\end{tabular}

* $P C N=$ parcela com cobertura natural; $P S D=$ parcela com condição de solo descoberto; $P C M=$ parcela com cobertura morta; $P F=$ parcela com cordoes vegetativos de Palma Forrageira.

** As letras indicam diferença significativa entre as médias, segundo o Teste de Tukey com 5\% de significância. 
A PCM e PF apresentaram maiores médias em todos os conjuntos avaliados, em relação a PSD, promovendo um acréscimo no conteúdo de água no solo. A cobertura natural promoveu em média, um incremento de $11 \%$ do conteúdo de água no solo, quando comparada a PSD. As práticas conservacionistas destacam-se por favorecer maior aproveitamento das águas das chuvas, evitando-se perdas excessivas por escoamento superficial e aumentando o tempo de contato da água com o solo, o que favorece a infiltração. Esses resultados também foram verificados por Lopes et al. (2019), avaliando no período de outubro de 2016 a outubro de 2017, no mesmo sítio experimental.
A Figura 5 apresenta os box-plots da umidade para os tratamentos e profundidades. Os valores correspondem à média dos 24 monitoramentos realizados. O traço interno é referente ao valor da mediana.

Observa-se elevadas umidades na camada de 0,2-0,4 m, com redução de aproximadamente $40 \%$ para a camada de $0-0,2 \mathrm{~m}$, corroborando com resultados de Borges et al. (2014) e Santos et al. (2010), que atribuíram esse comportamento ao maior teor de argila, promovendo maior retenção de água no solo em maior período de tempo. Nota-se também presença de valores atípicos, tanto na estação chuvosa quanto na estação seca. As parcelas sob Argissolo Amarelo (Malaquias e Edivaldo) apresentam

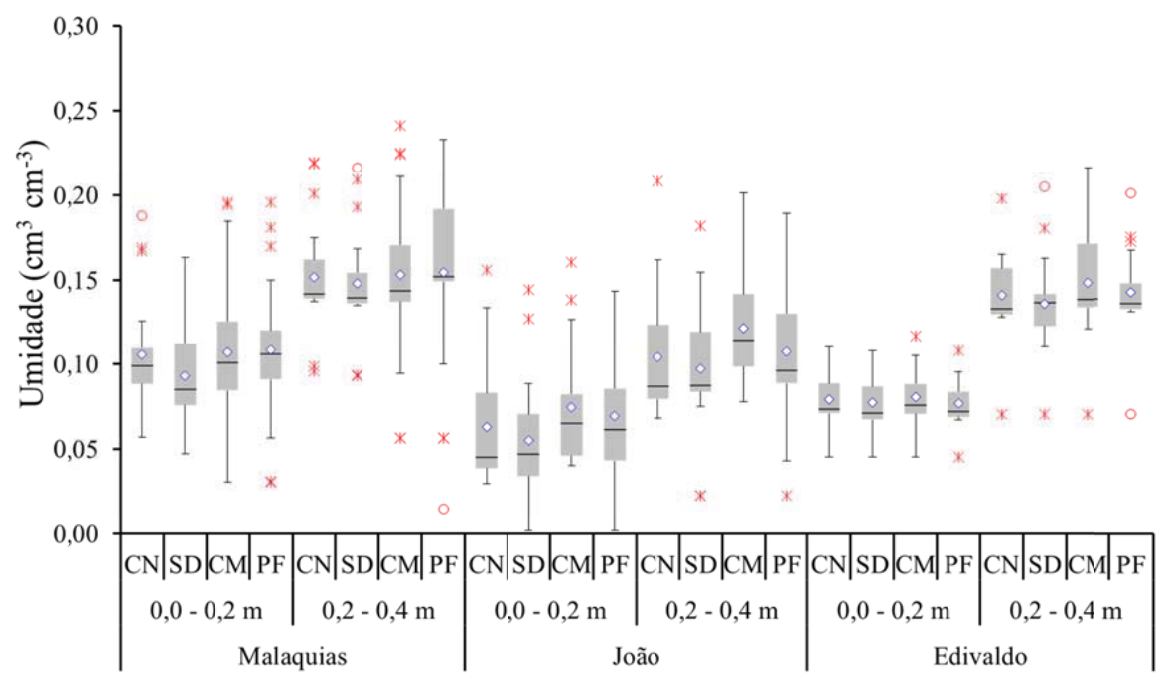

Figura 5- Distribuição estatística das umidades do solo por sítio experimental e tratamento, para as camadas de 0-0,20 m e 0,20-0,40 m.

Figure 5- Statistical distribution of soil moisture by experimental site and treatment, for the 0-0.20 $m$ and 0.20-0.40 m layers. 
maiores umidades em função da sua maior capacidade de retenção quando comparados com Neossolo Regolítico (Figura 5), corroborando com Melo e Montenegro (2015).

A geração de escoamento superficial, assim como as perdas de solo foram significativamente baixas para a condição PCN. Quando avaliadas as práticas conservacionistas de PCM e PF, estas reduziram eficientemente as perdas de água e solo, conforme o esperado. $\mathrm{O}$ escoamento apresentou comportamento diferente, com a PCN gerando a menor lâmina de escoamento e a condição PSD produzindo o maior escoamento, como observado na Figura 6, em que a PCN apresentou no máximo $30 \%$ de escoamento do que PSD.

As relações entre PSD e as condições conservacionistas permitem observar o quanto é importante o uso destas alternativas (Figura 7). Foi demonstrado que a PCM protegeu de forma eficiente a superfí- cie do solo nos três locais estudados, o que possibilitou maiores taxas de infiltração e consequentemente maior armazenamento de água no solo. Para PF, comportamento semelhante foi observado (Figura 7C), sendo que a perda máxima de solo foi de aproximadamente $49 \%$ da condição de solo descoberto. A PCM reduziu a perda de solo devido à formação de pequenas barreiras formadas pelo material em contato com o solo, dessa forma ocorrendo formação de pequenos contornos contra o fluxo de água.

Os valores de perda de solo em relação a condição de solo descoberto decresceram na seguinte sequência $\mathrm{PF}>\mathrm{PCM}>\mathrm{PCN}$. Brasil et al. (2017) associam essas condições à redução da energia cinética das chuvas e ao aumento do armazenamento de água no solo, possibilitando à $\mathrm{PCN}$ aumentar a infiltração e reduzir a evapotranspiração. Em ambas análises, a PCN de Caatinga foi eficiente em reduzir a perda de água e

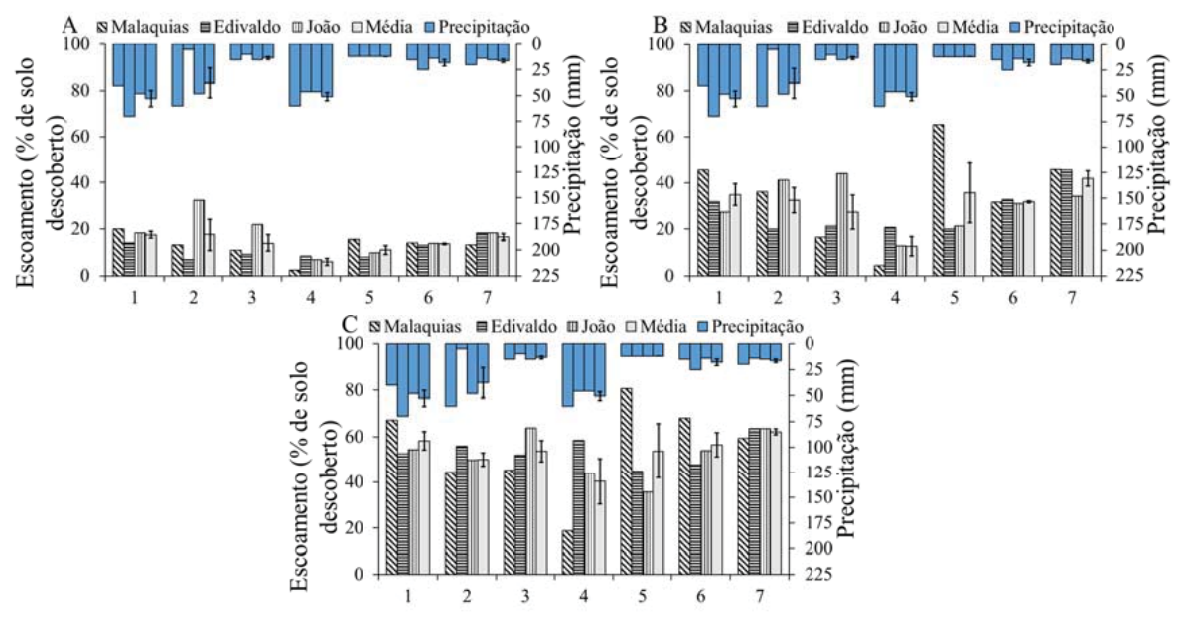

Figura 6- Eventos de escoamento superficial para (A) Cobertura; (B) Cobertura morta; (C) Palma. Figure 6- Runoff events for (A) Natural Cover; (B) Mulch Cover; (C) Palm. 
solo. Caloiero et al. (2016) ressaltam que apesar da presença de vegetação aumentar o consumo de água, a umidade do solo nas superfícies aumentou em comparação com as áreas onde a vegetação foi removida.
Observa-se na Tabela 2 as características hidráulicas do escoamento para a condição de solo descoberto (SD), cobertura morta de pó de coco a $8 \mathrm{Mg}^{-1} \mathrm{~h}^{-1}(\mathrm{CM})$ e cordão vegetal de Palma Forrageira (PF).

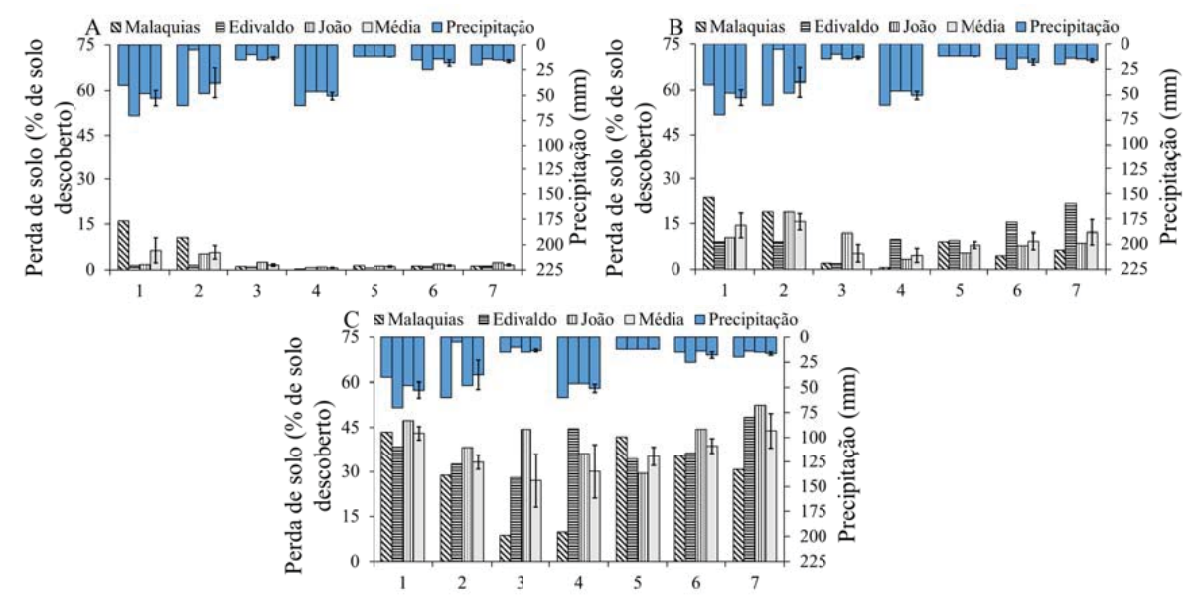

Figura 7- Perdas de solo nos eventos (A) Cobertura; (B) Cobertura morta; (C) Palma. Figure 7- Soil loss in the events for (A) Natural Cover; (B) Mulch Cover; (C) Palm.

Tabela 2. Parâmetros obtidos com simulações de chuva de $90 \mathrm{~mm} \mathrm{~h}^{-1}$, durante 15 minutos. Table 2. Parameters obtained fromrainfall simulations with $90 \mathrm{~mm} \mathrm{~h}^{-1}$, during 15 minutes.

\begin{tabular}{|c|c|c|c|}
\hline Variáveis & $\begin{array}{c}\text { Solo } \\
\text { Descoberto } \\
\text { (SD) }\end{array}$ & $\begin{array}{c}\text { Tratamentos } \\
\text { Solo com cobertura } \\
\text { morta } \\
(\mathrm{CM})\end{array}$ & $\begin{array}{c}\text { Palma } \\
\text { Forrageira } \\
\text { (PF) }\end{array}$ \\
\hline $\begin{array}{l}\text { Tempo para início do escoamento } \\
\text { (min) }\end{array}$ & 2,50 & 8,00 & 5,50 \\
\hline Volume escoado (I) & 11,00 & 4,20 & 7,40 \\
\hline Coeficiente de escoamento (\%) & 57,90 & 22,40 & 39,00 \\
\hline Perda de solo $(\mathrm{g})$ & 65,40 & 18,20 & 43,50 \\
\hline Umidade média inicial (\%) & 4,00 & 4,25 & 4,25 \\
\hline Umidade média final (\%) & 6,30 & 15,90 & 12,00 \\
\hline Número de Froude (adm.) & 0,45 & 0,17 & 0,18 \\
\hline Vel. do escoamento $\left(\mathrm{m} \mathrm{s}^{-1}\right)$ & 0,03 & 0,01 & 0,02 \\
\hline Lâmina altura do escoamento (mm) & 4,7 & 7,5 & 7,6 \\
\hline Coef. de rugosidade $\left(\mathrm{s} \mathrm{m}^{-1 / 3}\right)$ & 0,07 & 0,23 & 0,18 \\
\hline
\end{tabular}


A.A.A. Montenegro et al.

Para a condição de solo descoberto, o redução do escoamento, erosão hídrica e escoamento superficial se iniciou aos 2 aumento da umidade do solo. Quando comminutos e 30 segundos de chuva, com um parada ao solo descoberto, a cobertura morvolume total precipitado de 191 e atingin- ta apresentou um aumento significativo da do uma perda de solo e água de 65,4 g e infiltração da água no solo em consequên111 respectivamente. Santos et al. (2009) cia do maior tempo de contato entre a água e Borges (2014) ressaltam que, na ausência retida na cobertura e o solo. No tratamento de cobertura superficial do solo, o impacto de Palma Forrageira, o incremento na umidireto das gotas de chuva faz com que as dade do solo foi em função do barramento partículas de solo se dispersem ocorrendo proporcionado pela morfologia vegetal da assim altos valores de perda de solo. Quan- Palma, que proporciona a diminuição da do comparado ao SD, a cobertura morta re- velocidade do escoamento e que permite tardou o início do escoamento, e reduziu em $72 \%$ a perda de solo e em $62 \%$ a perda de água. Tais resultados também foram encontrados por Lima et al. (2015b) em teste com práticas agrícolas conservacionistas no cultivo da mandioca, onde a cobertura morta foi eficiente. O cordão vegetativo de Palma promoveu, assim como CM, uma redução na perda de solo e água de $40 \%$ e $33 \%$, respectivamente. A velocidade de escoamento nos tratamentos conservacionistas (cobertura morta e barramento com Palma) foi menor em relação ao solo descoberto, o que pode ser atribuído à barreira física ao escoamento e ao aumento da rugosidade hidráulica do fluxo superficial, com consequente aumento da altura do escoamento. Embora a rugosidade tenha aumentado, o Número de Froude diminuiu pela ação de resistência ao escoamento promovida pela rugosidade.

A redução da velocidade de escoamento promove o aumento da taxa de infiltração e consequentemente o aumento no teor de água no solo. Montenegro et al. (2013) em solos de Coimbra, Portugal, com cobertura de palha, também verificaram que a cobertura morta é uma prática eficiente em reduzir os danos causados pela ação erosiva das gotas de chuva, proporcionando uma um fluxo superficial mais lento ao longo da parcela.

\section{CONCLUSÕES}

A aplicação da cobertura morta proporcionou a redução do escoamento superficial, resultando numa maior infiltração de água no solo, principalmente na estação seca. Assim, a utilização de cobertura natural apresenta destacada relevância na preservação da umidade do solo, principalmente nos eventos de chuva de maior magnitude.

O tratamento com cordão de Palma Forrageira apresentou igualmente bom desempenho, tanto no controle das perdas de solo por erosão hídrica quanto na redução de velocidade do escoamento, promovendo a infiltração da água no solo a montante do cordão, favorecendo assim o umedecimento.

Deste modo, as práticas conservacionistas abordadas têm relevância no tocante à sustentabilidade do ambiente semiárido, sendo viáveis devido à sua simples implementação e ao seu baixo custo envolvido. 
Impacto de métodos naturais para conservação de água

\section{AGRADECIMENTOS}

Os autores agradecem ao Conselho Nacional de Desenvolvimento Científico e Tecnológico-CNPq (Processos $\mathrm{n}^{\circ}$ 420.488/2018-9 e 308.890/2018-3), à Fundação de Amparo à Ciência e Tecnologia do Estado de Pernambuco-FACEPE, ao projeto Aliança para Gestão de Recursos Hídricos no Semiárido de Pernambuco (Consórcio UNIVERSITAS) financiado pela FACEPE (Processo No. APQ-0300-5.03/17), à CAPES (Coordenação de Aperfeiçoamento de Pessoal de Nível Superior), à Pró-Reitoria de Extensão e Cultura (PROExC), à Financiadora de Estudos e Projetos-FINEP do Brasil e da FCT - Fundação para a Ciência e a Tecnologia (através dos Projetos ASHMOB referência CENTRO-01-0145-FEDER-029351, MUSSELFLOW referência PTDC/BIA-EVL/29199/2017 e MEDWATERICE referência PRIMA/0006/2018, apoiado no contexto do Programa PRIMA - Partnership for Research and Innovation in the Mediterranean Area), de Portugal.

\section{REFERÊNCIAS BIBLIOGRÁFICAS}

Borges TKS, Montenegro, AAA, Santos TEM, Silva DD, Silva Junior VP. 2014. Influência de práticas conservacionistas na umidade do solo e no cultivo do milho (Zea mays L.), no semiárido nordestino. Revista Brasileira de Ciência do Solo. 38: 1862-1873. http://dx.doi. org/10.1590/S0100-06832014000600021

Brasil JB, Palácio HADQ, Araújo Neto JRD, Ribeiro Filho JC, Andrade EMD. 2017. Características das chuvas e interceptação vegetal no
Bioma Caatinga. Irriga. 22: 560-574. https:// doi.org/10.15809/irriga.2017v22n3p560-574

Chen L, Huang Z, Gong J, Fu, B, Huang Y. 2007. The effect of land cover/vegetation on soil water dynamic in the hilly area of the loess plateau, China. Catena. 70: 200-208. https://doi.org/10.1016/j.catena.2006.08.007

Caloiero T., Biondo C., Callegari G., Collalti A., Froio R., Maesano M., Matteucci G., Pellicone G, Veltri A. 2016. Results of a longterm study on an experimental watershed in southern Italy. Forum Geografic. 15: 55-65. https://doi.org/10.5775/fg.2016.067.s

Cerdà A, Rodrigo-Comino J, Giménez-Morera A, Keesstra SD. 2017. An economic, perception and biophysical approach to the use of oat straw as mulch in Mediterranean rainfed agriculture land. Ecological Engineering. 108 (part A): 162-171. https://doi.org/10.1016/j. ecoleng.2017.08.028.

Cogo NP. 1978. Uma contribuição à metodologia de estudo das perdas de erosão em condições de chuva natural. I. Sugestões gerais, medição dos volumes, amostragem e quantificação de solo e água da enxurrada. 1a aproximação. In: Encontro Nacional de Pesquisa sobre Conservação do Solo, 2, Passo Fundo, Empresa Brasileira de Pesquisa Agropecuária. 75-98.

Coutinho AP, Silva FB, Silva RO, Antonino ACD, Montenegro SMGL. 2010. Determinação de Equações de Chuvas Intensas para Municípios das Mesorregiões do Estado de Pernambuco com dados pluviométricos. In Anais do Simpósio de Recursos Hídricos do Nordeste, Fortaleza: 14.

Darkwah KA, Kwawu JD, Agyire-Tettey F, Sarpong DB. 2019. Assessment of the determinants that influence the adoption of sustainable soil and water conservation practices in Techiman Municipality of Ghana. International Soil and Water Conservation Research. 
A.A.A. Montenegro et al.

7: 248-257. https://doi.org/10.1016/j.iswcr.2019.04.003

Flores P, Ausilio A, Leone A, Poggi D, Quadrelli A, Solini I, Lopez M, Monti F, Catraro M. 2019. Efecto de diferentes mulchings sobre el Crecimiento de una plantación de durazneros (Prunus persicae 1.) Variedad opodepe En zavalla, Provincia de Santa Fe. Ciências Agrárias. 18(1):49:58. https://doi. org/10.14409/fa.v18i1.8389

Haddaway NR, Brown C, Eales J, Eggers, S, Josefsson J., Kronvang B, Randall NP, Uusi-Kämppä, J. 2018. The multifunctional roles of vegetated strips around and within agricultural fields. Environmental Evidence. 7(1), 1-14. https://doi.org/10.1186/s13750016-0067-6

Hou D, Bolan NS, Tsang DCW, Kirkham MB, O'Connor D. 2020. Sustainable soil use and management: An interdisciplinary and systematic approach. Science of The Total Environment. 729: 1-12. https://doi.org/10.1016/j. scitotenv.2020.138961

Jordán A, Zavala LM, Gil J. 2010. Effects of mulching on soil physical properties and runoff under semi-arid conditions in southern Spain. Catena. 81 (1): 77-85. https://doi.org/10.1016/j.catena.2010.01.007

Lima CA, Montenegro AAA, Santos TEM, Andrade EM, Monteiro ALN. 2015b. Práticas agrícolas no cultivo da mandioca e suas relações com o escoamento superficial, perdas de solo e água. Revista Ciência Agronômica. 46 (4): 697-706. https://doi. org/10.5935/1806-6690.20150056.

Lima CA, Montenegro AAA, Santos TEM, Monteiro ALN, Pereira EG, Santos LM, Machado, LS. 2014. Características hidráulicas do escoamento superficial sob diferentes intensidades de chuva e práticas agrícolas.
In Anais do VI Congresso Sobre Uso e Manejo do Solo, Recife, março. 1: 53-55.

Lima SMDS, Montenegro AAA, da Silva JRL, Silva VCIS, de Lima CA, De Assis FMCV. 2015a. Variação de água no solo no semiárido pernambucano. III Inovagri International Meeting. Fortaleza, Brasil.

Lopes I, Montenegro AAA, de Lima JLMP. 2019. Performance of Conservation Techniques for Semiarid Environments: Field Observations with Caatinga, Mulch, and Cactus Forage Palma. Water. 11: 1-15; https://doi. org/10.3390/w11040792

Menezes JAL, Santos TEM, Montenegro AAA, Silva JRL. 2013. Comportamento temporal da umidade do solo sob Caatinga e solo descoberto na Bacia Experimental do Jatobá, Pernambuco. Water Resources and Irrigation Management-WRIM. 2 (1): 45-51.

Montenegro AAA, Abrantes JRCBO, de Lima JLMP, Singh V, Santos TEM. 2013. Impact of Mulching on Soil and Water Dynamics under Intermittent Simulated Rainfall. Catena. 109: 139 - 149. http://dx.doi.org/10.1016/j. catena.2013.03.018.

Montenegro SMGL, Montenegro AAA. 2004. Aproveitamento sustentável de aquíferos aluviais no semiárido. In: Cabral JJS, Ferreira JPCL, Montenegro SMGL, Costa W (Eds.). Água subterrânea: aquíferos costeiros e aluviões, vulnerabilidade e aproveitamento. Recife: Ed. Universitária da UFPE. Capítulo 2(1): 61-117.

Montenegro AAA, Lopes I, Carvalho AA, de Lima JLMP, Souza TEMS, Araújo HL, Lins FAC, Almeida TAB, Montenegro HCLA. 2019. Spatio Temporal Soil Moisture Dynamics and Runoff under Different Soil Cover Conditions in a Semiarid Representative Basin in Brazil. Advances in Geosciences. 
48(19): 19-30. https://doi.org/10.5194/adgeo-48-19-2019

Pan D, Gao X, Wang J, Yang M, Wu P, Dyck JM Zhao X. 2018. Vegetative filter strips-Effect of vegetation type and shape of strip on run off and sediment trapping. Land Degradation Development. 29:3917-3927. https://doi. org/10.1002/ldr.3160

Prats AS, Abrantes JR, Crema IP, Keizer JJ, de Lima JLMP. 2017. Runoff and soil erosion mitigation with sieved forest residue mulch strips under controlled laboratory conditions. Forest Ecology and Management, 396 102112; doi:10.1016/j.foreco.2017.04.019

Santos TEM; Montenegro AAA, Silva Junior VP, Montenegro SMGL. 2008. Erosão hídrica e perda de carbono orgânico em diferentes tipos de cobertura do solo no semiárido, em condições de chuva simulada. Revista Brasileira de Recursos Hídricos. 13: 29-34. https:// doi.org/ 10.21168/rbrh.v13n2.p113-125

Santos TEM, Montenegro AAA, Silva Júnior VP. 2009. Características hidráulicas e perdas de solo e água sob cultivo do feijoeiro no semiárido. Revista Brasileira de Engenharia Agrícola e Ambiental. 13: 217- 225. https://doi.org/10.1590/S141543662009000300001

Santos TEM, Silva DD, Montenegro AAA. 2010. Temporal variability of soil water content under different surface conditions in the semiarid region of Pernambuco State. Revista Brasileira de Ciência do Solo. 34:
1733-1741. https://doi.org/10.1590/S010006832010000500025.

Sarkar S, Singh SR. 2007. Interactive effect of tillage depth and mulch on soil temperature, productivity and water use pattern of rainfed barley (Hordium vulgare L.). Soil and Tillage Research. 92: 79-86. https://doi.org/10.1016/j.still.2006.01.014.

Shukla PR, Skea J, Slade R, Van Diemen R, Haughey E, Malley J, Pathak M, Pereira JP. (eds.) Technical Summary. 2019. In: Climate Change and Land: an IPCC special report on climate change, desertification, land degradation, sustainable land management, food security, and greenhouse gas fluxes in terrestrial ecosystems.

Varela A, Batista I, Madeira AC, Madeira M. 2014. Impacto das técnicas de conservação do solo e da água na produção agrícola e no solo da bacia da Ribeira Seca, Santiago. Cabo Verde - Agronomia e Recursos Naturais, ISAPRess Lisboa Editor: F Cardoso Pinto. pp.127-164. 